\title{
Planar Configurations of Lattice Vectors and GKZ-Rational Toric Fourfolds in $\mathbb{P}^{6}$
}

EDUARDO CATTANI

cattani@math.umass.edu

Department of Mathematics and Statistics, University of Massachusetts, Amherst, MA 01003, USA

ALICIA DICKENSTEIN

alidick@dm.uba.ar

Departamento de Matematica, FCEyN, Universidad de Buenos Aires, (1428) Buenos Aires, Argentina

Received April 10, 2002; Revised January 21, 2003; Accepted February 28, 2003

\begin{abstract}
We introduce a notion of balanced configurations of vectors. This is motivated by the study of rational $A$-hypergeometric functions in the sense of Gelfand, Kapranov and Zelevinsky. We classify balanced configurations of seven plane vectors up to $G L(2, \mathbb{R})$-equivalence and deduce that the only gkz-rational toric four-folds in $\mathbb{P}^{6}$ are those varieties associated with an essential Cayley configuration. We show that in this case, all rational $A$-hypergeometric functions may be described in terms of toric residues. This follows from studying a suitable hyperplane arrangement.
\end{abstract}

Keywords: $A$-hypergeometric functions, toric residues, balanced configurations, Cayley configurations

AMS Subject Classification: Primary 33C70, Secondary 05B35, 32A27

\section{Introduction}

A configuration $\left\{b_{1}, \ldots, b_{n}\right\}$ of vectors in $\mathbb{R}^{m}$ is said to be balanced if for every index set $J=\left\{1 \leq j_{1}<\cdots<j_{m-1} \leq n\right\}$, the multiset

$$
\left\{\operatorname{det}\left(b_{j_{1}}, \ldots, b_{j_{m-1}}, b_{i}\right) ; i \notin J\right\}
$$

is symmetric around the origin.

Balanced configurations in the plane with at most six vectors have been classified in [5]. Here we consider the case of seven planar vectors and show in Corollary 2.9 that there are only five balanced configurations up to $G L(2, \mathbb{R})$-equivalence. When the configuration is uniform, i.e. every pair of vectors is linearly independent, it is equivalent to a regular heptagon. In fact, if an arbitrary uniform configuration of plane vectors is balanced, then it is $G L(2, \mathbb{R})$-equivalent to a regular $(2 k+1)$-gon. This result was conjectured in the preprint version of this paper and recently verified by Ressayre [14].

E. Cattani is partially supported by NSF Grant DMS-0099707, and A. Dickenstein is partially supported by UBACYT X052 and ANPCYT 03-6568, Argentina. 
Our interest in balanced configurations stems from their relationship with multivariable hypergeometric functions in the sense of Gel'fand et al. [10, 12]. These functions are solutions of a regular, holonomic system of partial differential equations associated with a configuration $A \subset \mathbb{Z}^{d}$ and a homogeneity vector $\alpha \in \mathbb{C}^{d}$. They include, as particular examples, the classical Gauss hypergeometric function, as well as the multivariable generalizations of Appell, Horn, and Lauricella [9].

The combinatorics of the configuration $A$ plays a central role in the study of the $A$-hypergeometric system: from the construction of series solutions associated with regular triangulations of the convex hull of $A$ [12], to the recent Gröbner deformation methods of Saito et al. [16]. Conversely, the existence of rational solutions imposes strong combinatorial restrictions on $A$.

For appropriate integer homogeneities, every configuration $A$ admits polynomial solutions; indeed, they are related to the associated integer programming problem [15]. Similarly, for every $A$ and suitable $\alpha$, there exist Laurent polynomial solutions and, for projective curves, their number reflects algebraic properties of the toric ideal associated with $A$ [3]. We should also mention in this context, the classical work of Hermann Schwarz on algebraic solutions of Gauss' hypergeometric equation [13, Section 10.3].

In joint work with Bernd Sturmfels [5, 6], we have considered the combinatorial restrictions on $A$ imposed by the existence of $A$-hypergeometric rational functions other than Laurent polynomials. It is well-known that the irreducible components of the singular locus of the $A$-hypergeometric system are defined by facial discriminants, i.e. the sparse discriminant of a subconfiguration given by the intersection of $A$ with a face of its convex hull $[11,12]$. In particular, such discriminants are the possible factors of the denominator of a rational $A$-hypergeometric function. Following [5] we say that $A$ is gkz-rational if the discriminant $D_{A}$ is not a monomial and there exists a (non-zero) rational $A$-hypergeometric function $f$ whose denominator is a multiple of $D_{A}$. It is conjectured in [5, Conjecture 1.3] that a configuration is gkz-rational if and only if it is affinely equivalent to an essential Cayley configuration (c.f. (3.1)). This conjecture has been verified in [5] when the projective toric variety associated with $A$ is a hypersurface or has dimension at most three. Indeed, in the hypersurface case there is a direct correspondence between gkz-rationality and balanced configurations. Let $A$ be a $d \times(d+1)$-matrix of rank $d$ and $\left(b_{1}, \ldots, b_{d+1}\right)$ a $\mathbb{Z}$-generator of the integral kernel of $A$. Then [5, Theorem 2.3] asserts that $A$ is gkz-rational if and only if the multiset $\left\{b_{1}, \ldots, b_{d+1}\right\}$ is symmetric around the origin.

In this paper we study the first open case, that of codimension-two toric subvarieties of $\mathbb{P}^{6}$, in order to reveal the combinatorial and analytic problems that arise. Our starting point are the results in [8] about the sparse discriminant of codimension-two configurations. They allow us to show that in a gkz-configuration every non-splitting circuit (cf. Definition 2.4) must be balanced. Via Gale duality we are then led to the classification of balanced configurations of seven lattice vectors in the plane.

In Section 3 we deduce from the classification Theorem 2.8 that Conjecture 1.3 in [5] holds for toric four-folds in $\mathbb{P}^{6}$. That leads naturally to the question of describing the space of $A$-hypergeometric rational functions associated with the corresponding essential Cayley configuration. We show that, as predicted by [5, Conjecture 5.7], a suitable derivative of such a function can be realized as a toric residue in the sense of [2, 7]. As in [6], this is done 
by studying an appropriate oriented hyperplane arrangement. We conclude the paper with an example illustrating these constructions for the configuration associated with Appell's classical function $F_{2}$.

\section{Classification of two dimensional configurations}

Let $A=\left\{a_{1}, \ldots, a_{n}\right\} \subset \mathbb{Z}^{d}$, be an integral configuration spanning $\mathbb{Q}^{d}$. We denote by $m:=n-d$ the codimension of $A$. Let $B \in \mathbb{Z}^{n \times m}$ be a matrix, well defined up to right multiplication by elements of $G L(m, \mathbb{Z})$, whose columns $v_{1}, \ldots, v_{m}$ are a $\mathbb{Z}$-basis of the lattice

$$
\mathcal{L}:=\left\{v \in \mathbb{Z}^{n}: A \cdot v=0\right\}
$$

Let $b_{i}=\left(b_{i 1}, \ldots, b_{i m}\right) \in \mathbb{Z}^{m}$ be the $i$-th row of $B$. We shall also denote by $B$ the configuration $\left\{b_{1}, \ldots, b_{n}\right\} \in \mathbb{Z}^{m}$ and refer to it as a Gale dual of $A$.

Note that the vector $(1, \ldots, 1)$ is in the row span of $A$ if and only if

$$
\sum_{i=1}^{n} b_{i}=0 .
$$

Configurations satisfying (2.2) are called nonconfluent. This terminology will be explained in Section 3 when we discuss the hypergeometric system associated with a configuration.

Since in this paper we are ultimately interested in studying rational bivariate $A$ - hypergeometric functions we shall assume from now on that $A$ is a nonconfluent codimension-two configuration. We also make, without loss of generality, two simplifying assumptions:

Assumption 2.1 No hyperplane contains all but one point of $A$. Equivalently, all $b_{i} \neq 0$.

When this assumption is not satisfied, the discriminant $D_{A}=1$, and so $A$ cannot be gkz-rational.

Assumption 2.2 The configuration $A$ consists of distinct points. Dually, no line contains all but two of the vectors in $B$. In particular, we may assume from now on that $n \geq 4$.

In fact, the study of $A$-hypergeometric functions associated to configurations with repeated points reduces trivially to the study of hypergeometric functions associated to the configuration of the distinct points in $A$.

Given an index set $I \subset\{1, \ldots, n\}$, let $A(I)$ denote the subset $A(I)=\left\{a_{i} \in A ; i \in I\right\}$. Recall that a circuit in $A$ is a minimally dependent subset of $A$. A circuit $A(I)$ defines a codimension-one configuration so its Gale dual is given by a multiset $\left\{c_{i}, i \in I\right\} \subset \mathbb{Z} \backslash\{0\}$, well defined up to multiplication by -1 . These scalars define a $\mathbb{Z}$-minimal relation

$$
\sum_{i \in I} c_{i} a_{i}=0
$$


The vector $c \in \mathbb{Z}^{n}$ whose $i$-th component is $c_{i}$ and whose $j$-th component vanishes for all $j \in I^{c}$, belongs to $\mathcal{L}$ and, consequently, may be written as $c=\gamma_{1} v_{1}+\gamma_{2} v_{2}$. This means that the vectors $\left\{b_{j}, j \in I^{c}\right\}$ lie in the line orthogonal to $\left(\gamma_{1}, \gamma_{2}\right) \in \mathbb{Z}^{2}$. A subset $B(J)=\left\{b_{j} ; j \in J\right\}$ is a cocircuit if and only if it consists of all vectors of $B$ in the same line through the origin. Clearly, $B(J)$ is a cocircuit if and only if $A\left(J^{c}\right)$ is a circuit. In this case, given $j \in J$, the multiset

$$
\left\{\operatorname{det}\left(b_{i}, b_{j}\right) ; i \notin J\right\}
$$

is well defined up to multiplication by a non-zero constant and agrees, again up to constant, with the Gale dual of the circuit $A\left(J^{c}\right)$. In particular, the following definition is independent of the choice of $j \in J$.

Definition 2.3 A cocircuit $B(J)$ is said to be balanced if for some (all) $j \in J$, the multiset $\left\{\operatorname{det}\left(b_{i}, b_{j}\right) ; i \notin J\right\}$ is symmetric around the origin. $B$ is said to be balanced if all its cocircuits are balanced. Also, a circuit $A(I)$ is balanced if $B\left(I^{c}\right)$ is so or, equivalently, if its Gale dual is symmetric around the origin.

A fundamental property of Gale duality (cf. [1, Proposition 9.1.5]) states that the convex hull of a subset $A(I)$ is a face of the convex hull of $A$ if and only if the origin lies in the relative interior of $\operatorname{conv}\left(B\left(I^{c}\right)\right)$. Hence, in the codimension two case, a circuit $A(I)$ is contained in a face of $\operatorname{conv}(A)$ if and only if the cocircuit $B\left(I^{c}\right)$ contains at least two vectors of $B$ in opposite rays. Moreover, if $B(J)$ is a cocircuit and

$$
\sum_{j \in J} b_{j}=0
$$

the configuration $A$ can be written as $A=A(J) \cup A\left(J^{c}\right)$ with $A(J)$ and $A\left(J^{c}\right)$ lying in parallel flats.

Definition 2.4 A cocircuit $B(J)$ is splitting if equality (2.4) holds. Otherwise we call $B(J)$ non-splitting. We apply the same terminology to the corresponding circuit $A\left(J^{c}\right)$.

As Lemma 3.2 shows, non-splitting circuits behave well relative to discriminants and specialization of variables. Moreover, Theorem 3.3 implies that in order to classify gkzrationality, we must study configurations all of whose non-splitting circuits are balanced.

We give now two definitions that will help us organize the arguments. Let $B \subset \mathbb{R}^{2}$ be a finite vector configuration.

Definition 2.5 We say that $B$ is uniform if for every pair $i \neq j$, the vectors $b_{i}, b_{j}$ are linearly independent.

Note that in a uniform configuration every cocircuit is non-splitting. We also observe that any cocircuit whose complement consists of an odd number of vectors, is automatically 
unbalanced. In particular, a uniform balanced configuration must necessarily consist of an odd number of points.

Definition 2.6 We say that $B$ is irreducible if it is not possible to write it as a disjoint union $B=B_{1} \cup B_{2}$, where $B_{1}, B_{2}$ are proper nonconfluent subconfigurations.

We make the following convention: each time we write $B$ as a union of subconfigurations, we assume that we are in the conditions of the previous definition, i.e., that the union is disjoint and the subconfigurations are proper and with zero sum. In particular, each subconfiguration contains at least two vectors.

Remark 2.7 If a configuration satisfies Assumption 2.1, all subconfigurations do as well. Assumption 2.2, on the contrary, is not hereditary.

For $4 \leq n \leq 6$, the geometric classification of two dimensional Gale configurations satisfying Assumptions 2.1 and 2.2 follows from the results in [5] which we now recall.

- If $n=4$, every configuration $B$ is irreducible and uniform and therefore every cocircuit is non-splitting and unbalanced.

- For $n=5$, [5, Lemma 4.2] implies that either

(a) $B$ is irreducible and contains a non-splitting unbalanced cocircuit, or

(b) $B$ is irreducible and all its cocircuits are balanced, in which case it is $G L(2, \mathbb{R})$-equivalent to the regular pentagon, or

(c) $B$ is reducible; hence it is the union of a two-vector configuration $B_{1}$ and a threevector configuration $B_{2}$ not contained in a line. If $B$ is integral then it is Gale dual to an essential Cayley configuration (3.1).

- Suppose $n=6$. Then we have the following possibilities:

(a) $B$ contains a non-splitting unbalanced cocircuit, or

(b) $B$ is $G L(2, \mathbb{R})$-equivalent to $B_{1} \cup B_{2}$, where

$$
B_{i}=\left\{\lambda_{i} e_{1}, \lambda_{i} e_{2},-\lambda_{i} e_{1}-\lambda_{i} e_{2}\right\},
$$

and $\lambda_{1}+\lambda_{2} \neq 0$. In this case, all cocircuits are balanced.

(c) $B$ is a reducible configuration of the form $B=B_{1} \cup B_{2} \cup B_{3}$ or $B=B_{1} \cup B_{2}$ and each $B_{i}$ is a cocircuit.

The following is the main theorem in this section.

Theorem 2.8 Let $B=\left\{b_{1}, \ldots, b_{7}\right\} \subset \mathbb{R}^{2}$ be a nonconfluent configuration of seven non-zero vectors in the plane. Assume moreover that no line contains all but two of the vectors. Then, exactly one of the following holds:

(a) B contains a non-splitting unbalanced cocircuit.

(b) $B$ is irreducible and all its cocircuits are balanced, in which case it is $G L(2, \mathbb{R})$ equivalent to a regular heptagon. 
(c) $B$ is a reducible configuration of the form $B=B_{1} \cup B_{2} \cup B_{3}$ or $B=B_{1} \cup B_{2}$ with each $B_{i}$ a cocircuit.

(d) $B$ is a reducible configuration of the form $B=B_{1} \cup B_{2} \cup B_{3}$, with one $B_{i}$ having three vectors which generate $\mathbb{R}^{2}$.

(e) $B$ is a reducible configuration of the form $B=B_{1} \cup B_{2}$, where $B_{1}$ is a pair of opposite vectors and $B_{2}$ is $G L(2, \mathbb{R})$-equivalent to a regular pentagon.

Recall that an arbitrary configuration $B$ of planar vectors is called balanced if all its cocircuits are balanced.

Corollary 2.9 Let $B$ be a balanced configuration of seven non-zero vectors in the plane. Then $B$ is $G L(2, \mathbb{R})$-equivalent to one of the following:

(i) A regular heptagon,

(ii) a configuration $B=B_{1} \cup B_{2}$, where $B_{2}$ is a regular pentagon, and $B_{1}=\{\lambda c,-\lambda c\}$, $c \in B_{2}, \lambda \in \mathbb{R}^{*}$,

(iii) the configuration $B=\left\{e_{1}, e_{2},-e_{1}-e_{2}, \lambda e_{1},-\lambda e_{1}, \mu e_{2},-\mu e_{2}\right\}$, where $\lambda, \mu$ are nonzero scalars,

(iv) the configuration $B=\left\{e_{1}, e_{2},-e_{1}-e_{2}, \lambda e_{1},-\lambda e_{1}, \mu e_{1},-\mu e_{1}\right\}$, where $\lambda, \mu$ are non-zero scalars,

(v) a configuration of seven vectors in one line.

Proof: A configuration $B$ all of whose vectors are contained in a line is vacuously balanced. On the other hand, if $B$ spans the plane, then a balanced configuration is necessarily nonconfluent. If, in addition, $B$ satisfies Assumption 2.2 then it must be equivalent to one of the configurations (a)-(e) in Theorem 2.8. Of those, the configurations of type (a) and (c) cannot be balanced. If a configuration of type (e) is balanced then it must be as in (ii) since otherwise $B_{1}$ would be an unbalanced cocircuit.

Suppose now that $B$ is as in (d). Then, modulo $G L(2, \mathbb{R})$, we may assume that $B_{1}=$ $\left\{e_{1},-e_{1}\right\}, B_{2}=\left\{e_{2},-e_{2}\right\}$. Since the corresponding cocircuits must be balanced, this implies that $B_{3}=\left\{\lambda e_{1}, \mu e_{2},-\lambda e_{1}-\mu e_{2}\right\}$ which, after rescaling, yields the configuration (iii).

It remains to consider the case when a line contains at least five vectors of $B$. Clearly, since $B$ is nonconfluent, we must consider the case when all seven vectors lie in a line (case (v), or there are exactly five vectors in one line. If the other two vectors were also collinear then the corresponding cocircuit would be unbalanced. So we may assume that the remaining two vectors are linearly independent and that $B$ is $G L(2, \mathbb{R})$-equivalent to $B=\left\{\lambda_{1} e_{1}, \lambda_{2} e_{1}, \lambda_{3} e_{1}, \lambda_{4} e_{1}, \lambda_{5} e_{1}, \lambda_{6} e_{1}+\gamma e_{2}, e_{2}\right\}, \lambda_{6} \neq 0$. Condition 2.2 implies $\gamma=-1$, while the fact that the cocircuit defined by $e_{2}$ is balanced implies that, after reordering if necessary, $\lambda_{1}=-\lambda_{2}, \lambda_{3}=-\lambda_{4}$, and $\lambda_{5}=-\lambda_{6}$. Rescaling yields configuration (iv).

The proof of Theorem 2.8 will follow from a series of Lemmas. In their statements, (a)-(e) refer to the cases described in the theorem. Throughout, all configurations are supposed to verify Assumptions 2.1 and 2.2. Our first goal is to show that if $B$ is not uniform then either it contains a non-splitting unbalanced cocircuit or is as in (c), (d) or (e). 
Lemma 2.10 Suppose that $B$ is a reducible configuration such that $B=B_{1} \cup B_{2}$, where $B_{1}=\{b,-b\}$. Then $B$ is as in $(a),(c),(d)$ or $(e)$.

Proof: By Assumption 2.2, $B_{2}$ is a 5-vector configuration not contained in a line. Hence, either

- $B_{2}$ contains a non-splitting unbalanced cocircuit associated with a line $L$. Then $L$ also defines a non-splitting unbalanced cocircuit of the total configuration $B$, or

- $B_{2}$ is a reducible configuration which does not satisfy Assumption 2.2 and we are in case (c), or

- $B_{2}$ is a reducible configuration satisfying Assumption 2.2. Then so is $B$ and we are in case (d), or

- $B_{2}$ is equivalent to a regular pentagon and $B$ is as in (e).

Lemma 2.11 Suppose that there is a line $L$ containing an even number of vectors of $B$. Then $B$ is as in $(a),(c),(d)$, or $(e)$.

Proof: Since $L$ contains an even number of vectors, the associated cocircuit is unbalanced If it is non-splitting, then we are in case (a). Otherwise, the vectors in $B \cap L$ must add up to zero. If $L$ contains only two vectors then we are in the case covered by Lemma 2.10 . Hence we may assume that $L$ contains 4 vectors whose sum is zero. If the remaining 3 vectors lie on a line then we are in the case described by (c); otherwise, there is a line $L^{\prime}$ containing a single vector of $B$ and, consequently, the associated cocircuit is non-splitting. If it is unbalanced we are in case (a) while if it is balanced then necessarily there exist $b_{1}, b_{2} \in L$ such that $b_{1}=-b_{2}$ and we are again in the case covered by Lemma 2.10.

We can now restrict ourselves to configurations $B$ where each line contains an odd number of vectors of $B$. This means, in particular, that $B$ is distributed in an odd number of lines.

Lemma 2.12 Suppose that the configuration B is distributed in 3 lines, each containing an odd number of vectors. Then B contains a non-splitting unbalanced cocircuit, or it corresponds to cases $(c)$ or $(d)$.

Proof: By Assumption 2.2, no line may contain 5 vectors of $B$. Hence we need only consider the case when one of the lines, say $L_{1}$, contains only one vector, while the other two $L_{2}, L_{3}$ contain three vectors each. Let $Z_{i} \subset B$ denote the cocircuit associated with $L_{i}$. Because of Assumption 2.1, one of the cocircuits $Z_{2}, Z_{3}$ must be non-splitting. Assume $Z_{2}$ is non-splitting. If it is balanced, then there exist elements $b_{1}, b_{2} \in Z_{3}$ such that $b_{1}=-b_{2}$; hence we are in the case of Lemma 2.10 and, since $B$ is contained in three lines, it is as in (a), (c) or (d).

Lemma 2.13 Suppose that the configuration B is distributed in 5 lines, each containing an odd number of vectors. Then either B contains a non-splitting unbalanced cocircuit, 
or is as in $(d)$, or as in $(e)$ and the line containing $B_{1}$ contains one of the vertices of the pentagon.

Proof: It suffices to show that either $B$ contains a non-splitting unbalanced cocircuit or it reduces to the case in Lemma 2.10. Since one of the lines must contain three vectors of $B$, we may assume that $b_{1}=e_{1}, b_{2}=x e_{1}, b_{3}=y e_{1}$ with $x, y \neq-1$ and $x \neq-y$. We may also assume that $b_{4}=e_{2}$. Hence, if the cocircuit associated with the line $\mathbb{R} \cdot e_{2}$ is balanced, the configuration $B$ must be of the form:

$$
B^{t}=\left(\begin{array}{ccccccc}
1 & x & y & 0 & -1 & -x & -y \\
0 & 0 & 0 & 1 & * & * & *
\end{array}\right)
$$

If the cocircuit defined by $\mathbb{R} \cdot e_{1}$ is also balanced then either

$$
\begin{aligned}
B^{t} & =\left(\begin{array}{ccccccc}
1 & x & y & 0 & -1 & -x & -y \\
0 & 0 & 0 & 1 & -1 & u & -u
\end{array}\right), \quad u \neq 0, \text { or } \\
B^{t} & =\left(\begin{array}{lllllll}
1 & x & y & 0 & -1 & -x & -y \\
0 & 0 & 0 & 1 & u & -u & -1
\end{array}\right), \quad u \neq 0 .
\end{aligned}
$$

Consider the configuration (2.5). If the cocircuit associated with $\mathbb{R} \cdot(-1,-1)$ is balanced, we must have $u=y-x$. But then, the cocircuit defined by the line $\mathbb{R} \cdot(-x, u)=\mathbb{R} \cdot(-x, y-x)$ cannot be balanced since the multiset

$$
\{y-x, x(y-x), y(y-x), x,-y,-(y-x)(x+y)\}
$$

can never be symmetric around the origin given that $x, y \neq 0,-1$ since the configuration satisfies Assumption 2.1.

It remains to consider the case described by (2.6). It is easy to check that under our assumptions, if the cocircuit associated with the line $\mathbb{R} \cdot(-y,-u)$ is balanced then we must have $x=1+u y$. Consider then the cocircuit defined by $\mathbb{R} \cdot(-1, u)$. Checking again case-by-case we deduce that if it is balanced then $u=1+u y$ and hence $u=x$. We can then check that the last cocircuit, the one defined by $\mathbb{R} \cdot(-x, u)$, will be unbalanced unless $y=-x$.

Lemma 2.13 completes the proof of Theorem 2.8 for non-uniform configurations. The following result, was conjectured in the preprint version of this paper and proved for the case of seven plane vectors using Gröbner basis computations. Recently, Ressayre [14] gave a proof for the general case. For the sake of completeness, we include an adaptation of Ressayre's argument to our situation.

Theorem 2.14 Let $B$ be a uniform balanced configuration in the plane. Then $B$ is $G L(2, \mathbb{R})$-equivalent to a $(2 k+1)$-gon. 
Proof: We refer to [14] for the general case and sketch an argument in the case of seven vectors.

Let $\mathcal{P}$ denote the set of unordered pairs $\{(i, j): 1 \leq i, j \leq 7\}$ and

$$
\mathcal{P}^{k}=\left\{(i, j) \in \mathcal{P}: \operatorname{det}\left(b_{k}, b_{i}\right)=-\operatorname{det}\left(b_{k}, b_{j}\right)\right\} .
$$

Note that if $(i, j) \in \mathcal{P}^{k}$ then $b_{k}$ lies in the line spanned by $b_{i}+b_{j}$. Hence, the uniformity assumption implies that the sets $\mathcal{P}^{k}$ are disjoint. A cardinality argument shows that $\mathcal{P}=\bigcup_{k=1}^{7} \mathcal{P}^{k}$.

We also note that since every cocircuit is balanced, for each $k=1, \ldots, 7$, there exist three vectors $b_{i}$ such that $\operatorname{det}\left(b_{i}, b_{k}\right)>0$ and three vectors with negative determinant. Hence, each of the open hyperplanes determined by the line $\mathbb{R} \cdot b_{k}$ must contain exactly three vectors in the configuration. Suppose now that we index the vectors $b_{1}, \ldots, b_{7}$ counterclockwise, then the vector $b_{k}$ must be contained in the open cone spanned by $-b_{k-3}$ and $-b_{k+3}$, where we are indexing modulo 7 . This implies that $b_{k}$ is the only vector in the configuration that may lie in the line $\mathbb{R} \cdot\left(b_{k-3}+b_{k+3}\right)$ and, consequently,

$$
(k-3, k+3) \in \mathcal{P}^{k} .
$$

Similarly, we have

$$
(k-1, k+1) \in \mathcal{P}^{k} .
$$

Indeed, by symmetry it suffices to verify $(2.8)$ for the case $k=2$. Then $(1,3)$ must belong to either $\mathcal{P}^{2}$, since $b_{2}$ is in the open cone spanned by $b_{1}$ and $b_{3}$, or $\mathcal{P}^{5}$, or $\mathcal{P}^{6}$ since $b_{5}$ and $b_{6}$ are in the cone spanned by $-b_{1}$ and $-b_{3}$. However, it follows from $(2.7)$ that $(1,2) \in \mathcal{P}^{5}$ and $(2,3) \in \mathcal{P}^{6}$. Consequently, the last two cases are impossible and $(1,3) \in \mathcal{P}^{2}$, as claimed by (2.8). By a process of elimination we also have

$$
(k-2, k+2) \in \mathcal{P}^{k},
$$

and the set $\mathcal{P}^{k}$ is completely determined.

Modulo the action of $G L(2, \mathbb{R})$ we may assume that $b_{1}=e_{1}$ and $b_{2}=e_{2}$. Since $\mathcal{P}^{1}=$ $\{(2,7),(3,6),(4,5)\}$ and $\mathcal{P}^{2}=\{(1,3),(4,7),(5,6)\}$ the configuration $B$ must be of the form

$$
B^{t}=\left(\begin{array}{ccccccc}
1 & 0 & -1 & z & w & -w & -z \\
0 & 1 & x & y & -y & -x & -1
\end{array}\right)
$$

Since $(1,2) \in \mathcal{P}^{5}$ it follows that $b_{5}=\lambda\left(e_{1}+e_{2}\right)$ and, therefore, $w=-y$. Moreover, $(3,7) \in \mathcal{P}^{5}$ as well, so that $x=-z$. We also have that $(1,6) \in \mathcal{P}^{7}$ which implies that $y=x^{2}-1$. Therefore

$$
B^{t}=\left(\begin{array}{ccccccc}
1 & 0 & -1 & -x & 1-x^{2} & x^{2}-1 & x \\
0 & 1 & x & x^{2}-1 & 1-x^{2} & -x & -1
\end{array}\right) .
$$


Finally, since $(1,7) \in \mathcal{P}^{4}$ we have that

$$
x^{3}+x^{2}-2 x-1=0
$$

This equation has three real roots: $x=2 \cos (2 k \pi / 7), k=1,2,3$. A straightforward argument shows that the corresponding configurations are $G L(2, \mathbb{R})$-equivalent to a regular heptagon.

\section{Classification of rational hypergeometric functions}

We recall the definition of $A$-hypergeometric functions and refer to $[10,12,16]$ for their main properties.

Definition 3.1 Given an integer $d \times n$-matrix $A$ of rank $d$ and a vector $\alpha \in \mathbb{C}^{d}$, the A-hypergeometric system with parameter $\alpha$ is the left ideal $H_{A}(\alpha)$ in the Weyl algebra $\mathbb{C}\left\langle x_{1}, \ldots, x_{n}, \partial_{1}, \ldots, \partial_{n}\right\rangle$ generated by the toric operators $\partial^{u}-\partial^{v}$, for all $u, v \in \mathbb{N}^{n}$ such that $A \cdot u=A \cdot v$, and the Euler operators $\sum_{j=1}^{n} a_{i j} x_{j} \partial_{j}-\alpha_{i}$ for $i=1, \ldots, d$. A function $f\left(x_{1}, \ldots, x_{n}\right)$, holomorphic in an open set $U \subset \mathbb{C}^{n}$, is said to be A-hypergeometric of degree $\alpha$ if it is annihilated by $H_{A}(\alpha)$.

We say that the dimension of $A$ is $d-1$, i.e. the dimension of the affine span of its columns, and $m=n-d$ is its codimension. When $m=1$, the study of the hypergeometric system may be reduced to the study of a hypergeometric ordinary differential equation of degree $d$. The singularities of this equation are regular, and consequently the solutions have at worst logarithmic singularities, if and only if condition (2.2) is satisfied. Classically this is called the nonconfluent case. This terminology is extended to the multivariate situation. We shall assume throughout that the configuration $A$ is nonconfluent, which implies that the $A$-hypergeometric system is regular holonomic ([12, 16, 2.4.11]), and that it satisfies Assumptions 2.1 and 2.2. Since we are interested in the study of rational hypergeometric functions, we will also assume that $\alpha \in \mathbb{Z}^{d}$.

We recall that a configuration $A$ is said to be Cayley if $d=2 r+1$ and there exist vector configurations $A_{1}, \ldots, A_{r+1}$ in $\mathbb{Z}^{r}$ such that

$$
A=\left\{e_{1}\right\} \times A_{1} \cup \cdots \cup\left\{e_{r+1}\right\} \times A_{r+1} \subset \mathbb{Z}^{r+1} \times \mathbb{Z}^{r},
$$

where $e_{1}, \ldots, e_{r+1}$ is the standard basis of $\mathbb{Z}^{r+1}$. Moreover, $A$ is said to be essential if the Minkowski sum $\sum_{i \in I} A_{i}$ has affine dimension at least $|I|$ for every proper subset $I$ of $\{1, \ldots, r\}$.

If $A$ is a codimension-two Cayley configuration then the total number of points $n=2 r+3$ and, if $A$ is essential, each of the subsets $A_{i}$ must contain at least two points. Hence, in an essential Cayley configuration of codimension two, all but one of the $A_{i}$ 's contains two points and the remaining one contains three points. A generic sparse polynomial $f$ with 
support $A$ decomposes as

$$
f\left(s_{1}, \ldots, s_{r+1}, t_{1}, \ldots, t_{r}\right)=s_{1} f_{1}(t)+\cdots+s_{r+1} f_{r+1}(t),
$$

where $f_{1}, \ldots, f_{r}$ are binomials with respective supports $A_{1}, \ldots, A_{r}$ and $f_{r+1}$ is a trinomial with support $A_{r+1}$. Then, it is easy to deduce from [8, Section 5] that $A$ will be essential if and only if $D_{A} \neq 1$, and in this case $D_{A}$ equals the sparse resultant $R_{A_{1}, \ldots, A_{r+1}}\left(f_{1}, \ldots, f_{r+1}\right)$ [11].

Conjecture 1.3 in [5] asserts that the only gkz-rational configurations are those affinely isomorphic to an essential Cayley configuration. The codimension-one case as well as the dimension one, two, and three cases have been studied in [5]. In this section we verify this conjecture for the first significant open case: codimension-two configurations in dimension four, i.e. $n=7, d=5$. We prove, moreover, that for any such configuration the number of linearly independent stable rational hypergeometric functions (see Definition 3.5 below) is 1. In fact, a suitable derivative of any stable rational hypergeometric functions is a constant multiple of an explicit toric residue in the sense of $[2,7]$ associated to $A$ and the parameter vector $\alpha \in \mathbb{Z}^{5}$.

The following result generalizes, in the case of codimension-two configurations, Lemma 3.4 in [5].

Lemma 3.2 Let $A(I)$ be a non-splitting circuit of a codimension-two configuration A. Then there exists $j \in I^{c}$ such that a positive power of the discriminant $D_{A(I)}$ divides the specialization $\left.D_{A}\right|_{x_{j}=0}$.

Proof: We may assume without loss of generality that $I=\{1, \ldots, r\}$ and that for $j>r$, $b_{j}=\lambda_{j} e_{2}, \lambda_{j} \in \mathbb{Z}, \sum_{j>r} \lambda_{j}=\lambda>0$. This implies that $C\left(I^{c}\right)=\left\{b_{11}, \ldots, b_{r 1}\right\}$ and hence $D_{A(I)}$ is, up to an integer constant, the binomial

$$
\prod_{b_{i 1}>0} b_{i 1}^{b_{i 1}} \cdot \prod_{b_{i 1}<0} x_{i}^{-b_{i 1}}-\prod_{b_{i 1}<0} b_{i 1}^{-b_{i 1}} \cdot \prod_{b_{i 1}>0} x_{i}^{b_{i 1}},
$$

where the products run over $i \in I$. On the other hand, it follows from [8, Section 4] that the discriminant $D(A)$ may be computed, up to a monomial and integral factor, as the resultant (with respect to $t$ ) of two polynomials $p_{1}(t ; x), p_{2}(t ; x)$ with the following properties: $p_{1}(t ; x)$ does not involve the variables $x_{j}$, for $j>r, p_{1}(0 ; x)=D_{A(I)}$, and

$$
p_{2}(t ; x)=a_{1}(t) t^{\lambda} m_{1}(x)-a_{2}(t) m_{2}(x)
$$

with

$$
m_{1}(x)=\prod_{b_{k 2}<0} x_{k}^{-b_{k 2}}, \quad m_{2}(x)=\prod_{b_{k 2}>0} x_{k}^{b_{k 2}}
$$

where the products run over $k=1, \ldots, n$. 
By assumption, there exists $j>r$ such that $b_{j 2}=\lambda_{j}>0$. Hence, setting $x_{j}=0$ we obtain:

$$
\begin{aligned}
\left.D_{A}\right|_{x_{j}=0} & =\operatorname{Res}_{t}\left(\left.p_{1}(t ; x)\right|_{x_{j}=0},\left.p_{2}(t ; x)\right|_{x_{j}=0}\right) \\
& =\operatorname{Res}_{t}\left(p_{1}(t ; x), a_{1}(t) t^{\lambda} m_{1}(x)\right) .
\end{aligned}
$$

Poisson's formula for resultants now implies that the resultant

$$
\operatorname{Res}_{t}\left(p_{1}(t ; x), t^{\lambda}\right)=p_{1}(0 ; x)^{\lambda}=D_{A(I)}^{\lambda}
$$

divides (3.2).

Theorem 3.3 A codimension-two configuration which contains an unbalanced non-splitting circuit is not gkz-rational.

Proof: This result generalizes [5, Theorem 1.2] whose proof we follow. Let $A=\left\{a_{1}, \ldots\right.$, $\left.a_{n}\right\}$ be a gkz-rational configuration of codimension two. Suppose $f=P / Q$ is a rational $A$-hypergeometric function of degree $\alpha \in \mathbb{Z}^{d}$, where $P, Q \in \mathbb{C}\left[x_{1}, \ldots, x_{n}\right]$ are relatively prime. Assume, moreover, that $D_{A}$ is not a monomial and divides $Q$.

We claim that any non-splitting circuit $A(I)$ of $A$ is balanced. We may assume that $I=\{1, \ldots, r\}, r<n$ and, because of Lemma 3.2, that $\left.D_{A}\right|_{x_{n}=0}$ is not a monomial. Set $t=x_{n}, \tilde{A}=\left\{a_{1}, \ldots, a_{n-1}\right\}, \tilde{x}=\left(x_{1}, \ldots, x_{n-1}\right)$. We expand the $A$-hypergeometric function $f$ as

$$
f(\tilde{x} ; t)=\sum_{\ell \geq \ell_{0}} R_{\ell}(\tilde{x}) \cdot t^{\ell}
$$

where each $R_{\ell}(\tilde{x})$ is a rational $\tilde{A}$-hypergeometric function of degree $\alpha-\ell \cdot a_{n}$. Since $\left.D_{A}\right|_{x_{n}=0}$ is not a monomial, it follows from [5, Lemma 3.3] that some coefficient $R_{\ell}(\tilde{x})$ is not a Laurent polynomial. Hence, $\tilde{A}$ is gkz-rational. But $\tilde{A}$ is a codimension-one configuration; indeed, it is a pyramid over the circuit $A(I)$. Thus, it follows from [5, Theorem 2.3] that $A(I)$ must be balanced.

Theorem 3.4 A codimension two, dimension four, gkz-rational configuration is affinely equivalent to an essential Cayley configuration.

Proof: A Gale dual of $A$ is a configuration $B$ of seven lattice vectors in the plane. Hence, we may apply the classification given in Theorem 2.8. If $B$ is as in (a), it contains an unbalanced non-splitting circuit and then it may not be dual to a gkz-rational configuration by Theorem 3.3. A direct computation (see also [8, Corollary 4.5]) shows that the discriminant of any configuration $A$ whose Gale dual satisfies (c) must be 1 and hence $A$ is not gkzrational. Finally, note that no lattice configuration may be as in (b) or (e). 
We are left with Gale dual configurations $B=B_{1} \cup B_{2} \cup B_{3}$ as in (d). Two of the subconfigurations contain 2 vectors and the third has 3 vectors not on a line. Then, $A$ is Cayley essential and by [5, Theorem 1.5] it is gkz-rational.

We will now construct rational hypergeometric functions associated with an essential Cayley configuration. Let $A$ be Cayley essential with $r=2, n=7$. We may assume, modulo a change of coordinates, that the columns of $A$ index the coefficients in a sparse system of 3 equations of the form:

$$
\begin{aligned}
& f_{1}=x_{1}+y_{1} \cdot t_{1}^{\gamma_{1}} \\
& f_{2}=x_{2}+y_{2} \cdot t_{2}^{\gamma_{2}} \\
& f_{3}=x_{3}+y_{3} \cdot t_{1}^{\alpha_{1}} t_{2}^{\alpha_{2}}+z_{3} \cdot t_{1}^{\beta_{1}} t_{2}^{\beta_{2}},
\end{aligned}
$$

with $\gamma_{1}, \gamma_{2} \in \mathbb{Z}_{>0}$ and $\left(\alpha_{1}, \beta_{1}\right),\left(\alpha_{2}, \beta_{2}\right)$ non-zero integral vectors. For $i \neq j$, let $V_{i j}$ denote the common zero set of $f_{i}$ and $f_{j}$ in the torus $\left(\mathbb{C}^{*}\right)^{2}$. For any $a \in \mathbb{Z}^{2}, c \in \mathbb{Z}_{>0}^{3}$, the sum $R_{i j}(c, a)$ of the local residues at all the points in $V_{i j}$ of the differential 2-form

$$
\frac{t^{a}}{f_{1}^{c_{1}} f_{2}^{c_{2}} f_{3}^{c_{3}}} \frac{d t_{1}}{t_{1}} \wedge \frac{d t_{2}}{t_{2}}
$$

is a rational $A$-hypergeometric function of degree $(-c,-a)$. When $a$ lies in the interior of the Minkowski sum of the Newton polygons of $f_{1}^{c_{1}}, f_{2}^{c_{2}}$ and $f_{3}^{c_{3}}$, we have by Theorem 4.12 in [2] that

$$
R_{12}(c, a)=-R_{13}(c, a)=R_{23}(c, a) .
$$

Call this rational function $R(c, a)$. It has an integral representation [2, 17]:

$$
R(c, a)=\int_{\Gamma} \frac{t^{a}}{f_{1}^{c_{1}} f_{2}^{c_{2}} f_{3}^{c_{3}}} \frac{d t_{1}}{t_{1}} \wedge \frac{d t_{2}}{t_{2}}
$$

for an appropriate real 2-cycle $\Gamma$ in the torus.

As shown in [4, Theorem 7], we can differentiate $R(c, a)$ under the integral sign, for any $c \in \mathbb{Z}_{>0}^{3}, a \in \mathbb{Z}^{2}$. We have, for example,

$$
\begin{aligned}
& \partial_{x_{1}} R(c, a)=-c_{1} R(c+(1,0,0), a), \quad \text { and } \\
& \partial_{z_{3}} R(c, a)=-c_{3} R(c+(0,0,1), a+\beta) .
\end{aligned}
$$

Definition 3.5 A rational function $f$ is called unstable if it is annihilated by some iterated derivative. Otherwise we say that $f$ is stable.

Thus, a rational function $f$ is unstable if it is a linear combination of rational functions that depend polynomially on at least one of the variables. 
Proposition 3.6 Let $A$ be a Cayley essential configuration with $r=2, n=7$ and let $c \in \mathbb{Z}_{>0}^{3}$. Assume the integer vector $(c, a)$ lies in the integer image of $A$. Then, $R(c, a) \neq 0$. Moreover, $R(c, a)$ is stable.

Proof: We argue as in the proof of Proposition 4.4 in [6] which asserts a similar statement in case $f_{1}, f_{2}, f_{3}$ are binomials.

By definition, $R(c, a)$ may be computed as the sum of the local residues over $V_{12}$ of the form

$$
\frac{\left(t^{a} / f_{3}^{c_{3}}\right)}{f_{1}^{c_{1}} f_{2}^{c_{2}}} \frac{d t_{1}}{t_{1}} \wedge \frac{d t_{2}}{t_{2}}
$$

with respect to $\left(f_{1}, f_{2}\right)$. By the derivative formulae such as (3.6), it suffices to show the result for $c_{1}=c_{2}=c_{3}=1$. We may expand the numerator $t^{a} / f_{3}$ as a Laurent series of the form

$$
\frac{t^{a}}{f_{3}}=\frac{1}{x_{3}} \frac{t^{a}}{1+\left(y_{3} / x_{3}\right) t^{\alpha}+\left(z_{3} / x_{3}\right) t^{\beta}}=\sum_{m \in \mathbb{N}^{2}} c_{m} \frac{y_{3}^{m_{1}} z_{3}^{m_{2}}}{x_{3}^{m_{1}+m_{2}+1}} t^{a+m_{1} \alpha+m_{2} \beta},
$$

whith $c_{m} \neq 0$ for all $m=\left(m_{1}, m_{2}\right) \in \mathbb{N}^{2}$. By Lemma 4.2 in [6], the global residue with respect to $f_{1}, f_{2}$ of the form

$$
\frac{t^{a+m_{1} \alpha+m_{2} \beta}}{f_{1} \cdot f_{2}} \frac{d t_{1}}{t_{1}} \wedge \frac{d t_{2}}{t_{2}}
$$

vanishes unless there exists $v(m)=\left(v_{1}(m), v_{2}(m)\right) \in \mathbb{Z}^{2}$ such that

$$
a+m_{1} \alpha+m_{2} \beta=\left(v_{1}(m) \gamma_{1}, v_{2}(m) \gamma_{2}\right) \text {. }
$$

In this case the residue is equal (up to sign) to the Laurent monomial

$$
x_{1}^{\nu_{1}(m)-1} y_{1}^{-\nu_{1}(m)} x_{2}^{\nu_{2}(m)-1} y_{2}^{-\nu_{2}(m)} \text {. }
$$

Exchanging the residue integral and the sum of the series, we have

$$
R(c, a)=\sum \pm c_{m} \frac{y_{3}^{m_{1}} z_{3}^{m_{2}}}{x_{3}^{m_{1}+m_{2}+1}} x_{1}^{\nu_{1}(m)-1} y_{1}^{-v_{1}(m)} x_{2}^{\nu_{2}(m)-1} y_{2}^{-v_{2}(m)},
$$

where the sum runs over all $m \in \mathbb{N}^{2}$ for which the equation

$$
a+m_{1} \alpha+m_{2} \beta=\left(v_{1}(m) \gamma_{1}, v_{2}(m) \gamma_{2}\right)
$$

has an integral solution $v(m)$. 
Since $(c, a)$ lies in the integer image of $A,(3.8)$ has a solution for one, and a fortiori infinitely many pairs $\left(m_{1}, m_{2}\right)$. Moreover, the assumption that $\alpha$ and $\beta$ cannot be both multiples of $e_{1}$ or $e_{2}$ ensures that there will be infinitely many values of $v_{1}(m)$ and $v_{2}(m)$ as well. Hence, $R(c, a)$ and all its iterated derivatives are non zero.

Remark 3.7 We recall that the open cone in $\mathbb{R}^{5}$,

$$
\mathcal{E}=\left\{\sum_{i=1}^{7} v_{i} a_{i}: v_{i} \in \mathbb{R}, v_{i}<0\right\}
$$

is called the Euler-Jacobi cone of $A$. Then $(-c,-a)$ lies in $\mathcal{E}$ if and only if $c_{1}, c_{2}, c_{3}>0$ and $a$ lies in the interior of the Minkowski sum of the convex hulls of $c_{1} A_{1}, c_{2} A_{2}$ and $c_{3} A_{3}$ (i.e. the Newton polygons of $\left.f_{1}^{c_{1}}, f_{2}^{c_{2}}, f_{3}^{c_{3}}\right)$. Note that given $\alpha$ in the integer image of $A$, the vector $\alpha-A \cdot v$ lies in $\mathcal{E}$ for any integer vector $v \in \mathbb{N}^{n}$ with $v_{i} \gg 0$ for all $i=1, \ldots, n$.

Theorem 3.8 Let $A \subset \mathbb{Z}^{5 \times 7}$ be a Cayley essential configuration. Let $(-c,-a) \in \mathcal{E} \cap$ $A \cdot \mathbb{Z}^{7}$. Then, the dimension of the space of rational A-hypergeometric functions of degree $(-c,-a)$ is equal to 1 and it is spanned by $R(c, a)$.

Proof: By Proposition 3.6, it is enough to show that the dimension of the space of rational $A$-hypergeometric functions cannot exceed 1 . In order to prove this statement we need to recall the characterization of logarithm-free $A$-hypergeometric series of degree $(-c,-a)$. We refer to [16, Section 3.4] for details and proofs.

Let $B \in \mathbb{Z}^{7 \times 2}$ be a Gale dual of $A$; as before, we also denote by $B=\left\{b_{1}, \ldots, b_{7}\right\}$ the planar configuration of its row-vectors. Set $\mathcal{L}=\operatorname{ker}_{\mathbb{Z}}(A)$ and $\mathcal{L}_{\mathbb{R}}=\mathcal{L} \otimes_{\mathbb{Z}} \mathbb{R}$. For $v \in \mathbb{Z}^{7}$ such that $A \cdot v=(-c,-a)$, the plane $v+\mathcal{L}_{\mathbb{R}}$ may be identified with $\mathbb{R}^{2}$ via the affine isomorphism

$$
\lambda \in \mathbb{R}^{2} \mapsto v+B \cdot \lambda
$$

This correspondence maps $\mathbb{Z}^{2}$ to $v+\mathcal{L}$. Consider the affine arrangement of hyperplanes $\mathcal{H}$ in $\mathbb{R}^{2}$ given by

$$
H_{j}:=\left\{\lambda \in \mathbb{R}^{2}:\left\langle b_{j}, \lambda\right\rangle=-v_{j}\right\}, \quad j=1, \ldots, 7 .
$$

Each $H_{j}$ is oriented by the choice of normal vector $b_{j}$. The negative support of a vector $\lambda$ is defined as the set of all the indices $j=1, \ldots, 7$ for which $\left\langle b_{j}, \lambda\right\rangle<-v_{j}$. The (convex) set of points with the same negative support is called a cell of the hyperplane arrangement. A cell $\Sigma$ is minimal if $\Sigma \cap \mathbb{Z}^{2} \neq \emptyset$ and the negative support of the elements in this set is minimal with respect to inclusion among the supports of integer points in any other cell. If $(-c,-a) \in \mathcal{E}$ then all minimal cells are unbounded and correspond to minimal cells in the oriented central arrangement. To each minimal cell we may attach a formal Laurent 
series $\phi_{\Sigma}$, unique up to constant, whose exponents are the points in $\Sigma \cap \mathbb{Z}^{2}$, and which is annihilated by the action of the $A$-hypergeometric ideal $H_{A}((-c,-a))$.

Recall that we may assume that $B$ satisfies

$$
b_{1}+b_{2}=b_{3}+b_{4}=b_{5}+b_{6}+b_{7}=0 \text {. }
$$

The central arrangement defined by $b_{5}, b_{6}, b_{7}$ has three minimal cells. The lines orthogonal to $b_{1}, b_{3}$, may intersect at most two of those of cells, hence it is clear that there exists $w \in \mathbb{R}$ not parallel to any vector in $B$, for which there is a single minimal cell $\Sigma$ lying in a half-space $\langle w, \lambda\rangle>\rho$, for some $\rho \in \mathbb{R}$.

To complete the proof we need to show that the rational function $R(c, a)$ has a Laurent expansion with support in $\Sigma$. Moreover, since in the Euler-Jacobi cone there are no bounded minimal regions (cf. [6, Proposition 2.6]) it is enough to show that the linear functional $\langle w,$.$\rangle is bounded below in the preimage under (3.9) of the support of a Laurent expansion$ of $R(c, a)$.

Write $R(c, a)=P / Q$ with $P, Q$ polynomials without common factors. Since the discriminant $D_{A}$ divides $Q$, the Newton polytope of $Q, N(Q)$, must be two-dimensional. Moreover, since $Q$ is $A$-homogeneous, $N(Q)$ lies in a plane $\Pi$ parallel to $\mathcal{L}_{\mathbb{R}}$. Let $\tilde{w} \in \mathcal{L}_{\mathbb{R}}$ be the unique element such that $B \cdot \tilde{w}=w$ and choose $\mu_{0} \in \Pi$. Perturbing $w$ if necessary, we may assume that the linear functional

$$
\mu \in \Pi \mapsto\left\langle\tilde{w},\left(\mu-\mu_{0}\right)\right\rangle
$$

acting on $N(Q)$ attains its minimum only at a vertex $v_{0}$ of $N(Q)$. This is clearly independent of the choice of $\mu_{0}$, hence we may assume from now on that $\mu_{0}=v_{0}$. Let $v_{1}, \ldots, v_{a}$ denote the remaining integral points of $N(Q)$; note that, by construction,

$$
\left\langle\tilde{w},\left(v_{j}-v_{0}\right)\right\rangle>0 ; \quad j=1, \ldots, a
$$

If we expand $1 / Q$ at $v_{0}$ (cf. $\left.[12,6.1 . b]\right)$ we obtain

$$
1 / Q=x^{-v_{0}} \cdot \sum_{\alpha \in \mathbb{N}^{a}} c_{\alpha} x^{\sum \alpha_{j}\left(v_{j}-v_{0}\right)}
$$

Now, for any monomial $x^{u}$ in $P$, the exponents of $x^{u} / Q$ are of the form $\left(u-v_{0}\right)+\sum \alpha_{j}$ $\left(v_{j}-v_{0}\right)$ and clearly, $\langle w,$.$\rangle is bounded below in the preimage under (3.9) of this set.$ Finally, since the support of $P / Q$ is contained is a finite union of sets of this form, the result follows.

As a Corollary, we identify all stable rational functions for any integer homogeneity, proving in this case Conjecture 5.7 in [5].

Corollary 3.9 Let $\alpha \in \mathbb{Z}^{5}$. The dimension of the space of rational A-hypergeometric functions of degree $\alpha$, modulo unstable ones, is at most 1 . Moreover, for any rational 
A-hypergeometric function, an iterated derivative of it is zero or a multiple of a toric residue.

Proof: If there is a non-zero rational $A$-hypergeometric function $f$ of degree $\alpha$, then $\alpha$ is necessarily in the integer image of $A$. Moreover, there exists $v \in \mathbb{N}^{7}$ such that $\alpha-$ $A \cdot v \in \mathcal{E} \cap A \cdot \mathbb{Z}^{7}$. Hence, the derivative $D_{v}(f)$ is a multiple (possibly zero) of a toric residue.

Example 3.10 We recall that there are 14 complete hypergeometric Horn series in two variables and of order two [9, Section 5.7.1]. Modulo monomial changes of variables and analytic continuation, they give rise to 8 different functions [10, Section 3.2]. In combinatorial terms they correspond to Gale configurations in $\mathbb{Z}^{2}$ of vectors with the property that the sum of positive first or second entries is equal to two. In particular, there exists a unique such configuration of seven vectors and it is associated with the classical hypergeometric series $F_{2}, F_{3}$ and $H_{2}$. The configuration $A$ and a Gale dual $B$ can be chosen as:

$$
A=\left(\begin{array}{lllllll}
1 & 1 & 0 & 0 & 0 & 0 & 0 \\
0 & 0 & 1 & 1 & 0 & 0 & 0 \\
0 & 0 & 0 & 0 & 1 & 1 & 1 \\
0 & 1 & 0 & 0 & 0 & 1 & 0 \\
0 & 0 & 0 & 1 & 0 & 0 & 1
\end{array}\right) ; \quad B=\left(\begin{array}{rr}
1 & 0 \\
-1 & 0 \\
0 & 1 \\
0 & -1 \\
-1 & -1 \\
1 & 0 \\
0 & 1
\end{array}\right) .
$$

Thus, this is a Cayley essential configuration, with $A_{1}=\left\{0, e_{1}\right\}, A_{2}=\left\{0, e_{2}\right\}, A_{3}=$ $\left\{0, e_{1}, e_{2}\right\} \subset \mathbb{Z}^{2}$. Let $f_{1}(t), f_{2}(t), f_{3}(t)$ be as in $(3.4), c=(1,1,1)$, and $a=(0,0)$. Then $R_{12}(c, a)\left(x_{1}, \ldots, z_{3}\right)$ may be computed as a single local residue

$$
\begin{aligned}
R_{12}(c, a) & =\operatorname{Res}_{\left(-\frac{x_{1}}{y_{1}},-\frac{x_{2}}{y_{2}}\right)}\left(\frac{1 /\left(t_{1} t_{2}\left(x_{3}+y_{3} t_{1}+z_{3} t_{2}\right)\right)}{\left(x_{1}+y_{1} t_{1}\right)\left(x_{2}+y_{2} t_{2}\right)} d t_{1} \wedge d t_{2}\right) \\
& =\frac{y_{1} y_{2}}{x_{1} x_{2}\left(y_{1} y_{2} x_{3}-x_{1} y_{2} y_{3}-y_{1} x_{2} z_{3}\right)} .
\end{aligned}
$$

Although (3.5) does not hold in this degree, this is the unique, up to constant, stable rational $A$-hypergeometric function of degree $(-c,-a)$. The function $R_{12}(c, a)$ may also be written as

$$
R_{12}(c, a)=\frac{1}{x_{1} x_{2} x_{3}}\left(\frac{1}{1-u-v}\right) ; \quad u=\frac{x_{1} y_{3}}{y_{1} x_{3}}, \quad v=\frac{x_{2} z_{3}}{y_{2} x_{3}}
$$

and

$$
\frac{1}{1-u-v}=\sum_{m, n \geq 0}\left(\begin{array}{c}
m+n \\
m
\end{array}\right) u^{m} v^{n}=F_{2}\left(1, \beta, \beta^{\prime}, \beta, \beta^{\prime} ; u, v\right)
$$


for any $\beta, \beta^{\prime} \in \mathbb{C} \backslash \mathbb{Z}_{\leq 0}$. Note that the vector $(-1,-1,-1,0,0)$ does not lie in the EulerJacobi cone and, indeed, there are unstable rational functions of this degree. They are also realized as residues:

$$
\begin{aligned}
R_{1}\left(x_{1}, \ldots, z_{3}\right) & =\operatorname{Res}_{\left(-\frac{x_{1}}{y_{1}}, 0\right)}\left(\frac{1 /\left(t_{1} f_{2}(t) f_{3}(t)\right)}{t_{2}\left(x_{1}+y_{1} t_{1}\right)} d t_{1} \wedge d t_{2}\right) \\
& =\frac{y_{1}}{x_{1} x_{2}\left(y_{1} x_{3}-x_{1} y_{3}\right)}, \\
R_{2}\left(x_{1}, \ldots, z_{3}\right) & =\operatorname{Res}_{\left(0,-\frac{x_{2}}{y_{2}}\right)}\left(\frac{1 /\left(t_{2} f_{1}(t) f_{3}(t)\right)}{\left(x_{2}+y_{2} t_{2}\right) t_{1}} d t_{1} \wedge d t_{2}\right) \\
& =\frac{y_{2}}{x_{1} x_{2}\left(y_{2} x_{3}-x_{2} z_{3}\right)}, \text { and } \\
R_{3}\left(x_{1}, \ldots, z_{3}\right) & =\operatorname{Res}_{(0,0)}\left(\frac{1 /\left(f_{1}(t) f_{2}(t) f_{3}(t)\right)}{t_{1} t_{2}} d t_{1} \wedge d t_{2}\right) \\
& =\frac{1}{x_{1} x_{2} x_{3}} .
\end{aligned}
$$

These four rational functions are linearly independent and the holonomic rank of the hypergeometric system $H_{A}(-c,-a)$ is 4 , so in this example all solutions are rational, which is highly exceptional.

On the other hand, for $c=(1,1,2), a=(1,1)$, the vector $(-c,-a)$ is in $\mathcal{E}$ and there exists a unique, up to constant, rational $A$-hypergeometric function of this degree:

$$
\begin{aligned}
R(c, a) & =\operatorname{Res}_{\left(-\frac{x_{1}}{y_{1}},-\frac{x_{2}}{y_{2}}\right)}\left(\frac{1 /\left(x_{3}+y_{3} t_{1}+z_{3} t_{2}\right)^{2}}{\left(x_{1}+y_{1} t_{1}\right)\left(x_{2}+y_{2} t_{2}\right)} d t_{1} \wedge d t_{2}\right) \\
& =\frac{y_{1} y_{2}}{\left(y_{1} y_{2} x_{3}-x_{1} y_{2} y_{3}-y_{1} x_{2} z_{3}\right)^{2}} \\
& =\frac{1}{x_{3}^{2} y_{1} y_{2}} \cdot \frac{1}{(1-u-v)^{2}} \\
& =\frac{1}{x_{3}^{2} y_{1} y_{2}} \cdot F_{2}\left(2,1, \beta^{\prime}, 2, \beta^{\prime} ; u, v\right),
\end{aligned}
$$

for any parameter $\beta^{\prime} \in \mathbb{C} \backslash \mathbb{Z}_{\leq 0}$.

\section{Acknowledgments}

We are grateful to Bernd Sturmfels for many valuable discussions and to the two anonymous referees for their comments and suggestions for improvement. This work was completed while Alicia Dickenstein was visiting the University of Stockholm with the support of the Swedish Science Council. She is grateful to the Department of Mathematics and to Mikael Passare for their hospitality. 


\section{References}

1. A. Björner, M. Las Vergnas, B. Sturmfels, N. White, and G. Ziegler, Oriented Matroids, Cambridge University Press, 1993.

2. E. Cattani, D. Cox, and A. Dickenstein, "Residues in toric varieties," Compositio Mathematica 108 (1997), 35-76.

3. E. Cattani, C. D'Andrea, and A. Dickenstein, "The $\mathcal{A}$-hypergeometric system associated with a monomial curve," Duke Math. J. 99 (1999), 179-207.

4. E. Cattani and A. Dickenstein, "A global view of residues in the torus," Journal of Pure and Applied Algebra 117/118 (1997), 119-144.

5. E. Cattani, A. Dickenstein, and B. Sturmfels, "Rational hypergeometric functions," Compositio Mathematica 128 (2001), 217-240.

6. E. Cattani, A. Dickenstein, and B. Sturmfels, "Binomial Residues," Ann. Inst. Fourier 52 (2002), 687-708.

7. D. Cox, "Toric residues," Arkiv för Matematik 34 (1996), 73-96.

8. A. Dickenstein and B. Sturmfels, "Elimination theory in codimension two," Journal of Symbolic Computation 34 (2002), 119-135.

9. A. Erdélyi et al., Higher Transcendental Functions, Based, in part, on notes left by Harry Bateman, McGrawHill Book Company, Inc., New York-Toronto-London, 1955.

10. I.M. Gel'fand, M. Kapranov, and A. Zelevinsky, "Generalized Euler integrals and $\mathcal{A}$-hypergeometric functions," Advances in Math. 84 (1990), 255-271.

11. I.M. Gel'fand, M. Kapranov, and A. Zelevinsky, Discriminants, Resultants and Multidimensional Determinants, Birkhäuser, Boston, 1994.

12. I. M. Gel'fand, A. Zelevinsky, and M. Kapranov, "Hypergeometric functions and toral manifolds," Functional Analysis and its Appl. 23 (1989), 94-106.

13. E. Hille, Ordinary Differential Equations in the Complex Domain, Dover, N.Y., 1997.

14. N. Ressayre, "Balanced configurations of $2 n+1$ plane vectors," Preprint (2002), arXiv:math.RA/0206234.

15. M. Saito, B. Sturmfels, and N. Takayama, "Hypergeometric polynomials and integer programming," Compositio Mathematica 115 (1999), 185-204.

16. M. Saito, B. Sturmfels, and N. Takayama, Gröbner Deformations of Hypergeometric Differential Equations, Algorithms and Computation in Mathematics, Vol. 6, Springer-Verlag, Heidelberg, 1999.

17. A. Tsikh, Multidimensional Residues and Their Applications, American Math. Society, Providence, 1992. 